\title{
Effect of Structural Remodeling (Retraction and Recoil) of the Pipeline Embolization Device on Aneurysm Occlusion Rate
}

L.-D. Jou, B.D. Mitchell, H.M. Shaltoni, and M.E. Mawad

\begin{abstract}
BACKGROUND AND PURPOSE: During endovascular treatment of unruptured aneurysms with the Pipeline Embolization Device, an oversized device is often selected to achieve better wall apposition; however, this device oversizing could be related to overelongation and possible delayed enlargement of the stented region. The purpose of this study is to investigate the relationship between oversize and treatment outcome.
\end{abstract}

MATERIALS AND METHODS: The DynaCT images of 14 aneurysms treated by a single Pipeline Embolization Device were retrospectively analyzed. 3D images of the deployed device were compared with those acquired at the 6-month follow-up for qualitative and quantitative evaluation. The diameter and length of the Pipeline Embolization Device were measured at both time points and compared for determination of the device changes.

RESULTS: Structural changes of the device have been observed, and it was found that the Pipeline Embolization Device influences the vessel curvature in some cases. On average, it increases its diameter by $0.23 \mathrm{~mm}$ and decreases its length by $2.88 \mathrm{~mm}$ within 6 months of initial deployment. Excessive elongation beyond its nominal length is correlated with a lower aneurysm occlusion rate at the 6-month follow-up.

CONCLUSIONS: Not only does a Pipeline Embolization Device reconstruct the aneurysm and parent artery, but its entire structure goes through a gradual remodeling process. The relative deformation between the device and the artery indicates suboptimal wall apposition. Device oversizing does not have a direct effect on shortening or recoil. The aneurysm occlusion rate, however, is lowered by overelongation of the Pipeline Embolization Device.

ABBREVIATION: PED = Pipeline Embolization Device

G rowing enthusiasm for the Pipeline Embolization Device (PED; Covidien, Irvine, California) has prompted a dramatic shift in how unruptured aneurysms are managed since approval of the device. ${ }^{1}$ The PED diverts blood away from the aneurysm and reduces rupture risk by stimulating formation of intra-aneurysmal thrombus and lowering hemodynamic forces on the aneurysm dome.

Few aneurysms treated by flow diversion are thrombosed acutely; the aneurysm occlusion rate varies from center to center, but in general, it increases with time, from $68 \%$ at the 3 -month, ${ }^{2}$ $65 \%-91 \%$ at the 6-month, ${ }^{3,4} 90 \%-93 \%$ at the $12-$ month, ${ }^{5}$ and

Received October 18, 2012; accepted after revision January 6, 2014.

From the Departments of Radiology (L.-D.J., H.M.S., M.E.M.) and Neurosurgery (B.D.M.), Baylor College of Medicine, Houston, Texas.

Please address correspondence to Liang-Der Jou, PhD, Department of Radiology, Baylor College of Medicine, One Baylor Plaza, Houston, Texas 77030; e-mail: jou@bcm.tmc.edu

EIndicates article with supplemental on-line table.

Indicates article with supplemental on-line figure.

http://dx.doi.org/10.3174/ajnr.A3920
$84 \%$ at the 18 -month follow-up. ${ }^{6}$ Nevertheless, it is not uncommon for aneurysms to remain patent $>2$ years after treatment, and the risk of delayed hemorrhage persists. ${ }^{7,8}$

Intracranial stents are known to alter vessel curvature ${ }^{9}$; similarly, the parent artery may cause deformation of a flow diverter and influence the device porosity. ${ }^{10}$ Vessel tortuosity often renders a nonuniform distribution of the porosity and allows potentially less metal coverage at the aneurysm neck; as a result, prediction of the timing of complete aneurysm occlusion after flow diversion can be a challenge. The PED is a braided self-expanding stent with 12 platinum and 36 cobalt-chromium alloy strands. Radio-opacity of the platinum strands in a PED can be used as a marker and offers an opportunity to visualize the PED in greater detail to reveal suboptimal deployment, which may require further intervention. ${ }^{11,12}$ In this study, we investigated the temporal change of the PED structure in situ after deployment and examined its relationship with the aneurysm occlusion rate.

\section{MATERIALS AND METHODS}

This retrospective study was approved by the Human Subject Research Committee at our institution before commencement. 
Nineteen consecutive patients who received only 1 PED device for treatment of internal carotid artery aneurysms between June 2011 and March 2012 were included in the study. Five patients were excluded due to their age or lack of appropriate 3D images. The remaining 14 patients ( 12 women and 2 men) had a follow-up angiographic examination between 6 and 7 months. Three patients had additional examinations for treatment of other aneurysms before 6 months. Four aneurysms were at the ophthalmic; 4 , at the supraclinoid; and 2, at the cavernous segment. The remaining aneurysms were located at the anterior choroidal artery, superior hypophyseal artery, and the petrous or cervical segment of the ICA. Twelve aneurysms were saccular, and 2 were either dissected or dysplastic.

At each treatment, it was determined by the senior neuroradiologist that flow stasis was adequate after the first PED and an additional PED was not required. Patients who were treated by $>1$ PED during the same session were excluded from the study. After deployment, DynaCT images (Siemens, Erlangen, Germany) with and/or without contrast were acquired. ${ }^{13}$ These DynaCT images were reconstructed at 0.11 - to $0.13-\mathrm{mm}$ resolution so that each radio-opaque strand could be visualized and analyzed. The same procedure was repeated at the subsequent follow-up examination.

These patients were divided into 2 groups based on the condition of their aneurysms at the 6-month follow-up: 7 patients with completely occluded aneurysms (group 1) and 7 with incompletely obliterated aneurysms (group 2). The average age of these patients was 61 years ( 59 years for group 1 and 62 years for group 2 ), and the mean aneurysm size was $8.1 \mathrm{~mm}$ (6.2 $\mathrm{mm}$ for group 1 and $9.4 \mathrm{~mm}$ for group 2). Neither age nor aneurysm size was statistically different between the 2 groups. Two patients from group 1 and none in group 2 were smokers and hypertensive; these risk factors were not significantly different between the 2 groups.

\section{Qualitative Comparisons}

The DynaCT images of the PED acquired at different time points were compared on the Leonardo workstation (Siemens) by intensity thresholding and automatic coregistration. The coregistration was performed with the PED structure as the landmark after removal of all tissues. These comparisons revealed the deformation of each individual strand, and the change of the PED structure and general PED integrity were also scrutinized. The DynaCT images obtained with contrast also permitted evaluation of the relative position between the PED and aneurysm.

\section{Quantitative Measurements}

For each case, the proximal and distal circumferences of the PED device were measured on the Leonardo workstation. The circumferences were selected because the cross-section might not be circular, and a direct diameter measurement was prone to errors. The circumference was converted to an equivalent diameter so that a comparison of the size could be made. However, this comparison potentially disregarded any change in the shape of the cross-section.

Six lines consisting of intersection points of metal strands along the PED were constructed, and an example of measuring the PED length by using these lines is demonstrated in Fig 1. The

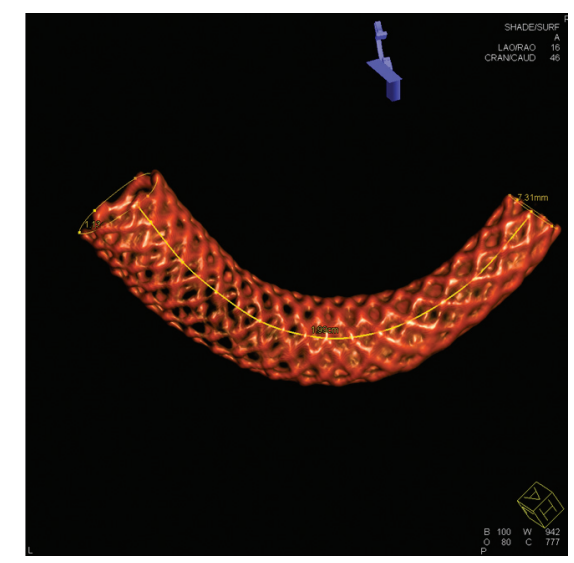

FIG 1. Demonstration of measurement of the PED length. Only 1 of the 6 material lines is shown.

intersection of metal strands yielded the highest signal intensity and could be easily depicted on the DynaCT images. These intersection points were physically present and could be connected by a simple interpolated spline to form a material line aligned in the same direction as the PED. The length measurement of a material line was superior to the measurement of the centerline because the centerline was an imaginary one, while the material line could be easily generated by connecting visible physical points. The process of generating a material line was independent of operators or numeric algorithms. Six material lines along the PED beginning from 6 different strands were generated, and the average length of these lines was regarded as the PED length. Usually, these material lines were of different lengths, with the lines on the outer curve longer.

Each measurement (PED circumference and length) was repeated for assessment of possible errors associated with measurement, and intermeasurement differences were no more than $2 \%$. The measurements were also compared with the nominal dimensions. The radius of the curvature was not measured because most PEDs had $>1$ curvature and a single curvature could not properly represent the entire device. The Student $t$ test was used for testing the statistical significance of these measurements. A $P$ value $<.05$ was deemed statistically significant.

\section{RESULTS}

At the 6-month follow-up, many PEDs were observed to retract at either the proximal or distal end (or both), making the PED shorter overall. However, this retraction was not related to any clinical consequences or symptoms; in addition, no migration of the PED was observed. ${ }^{14}$ In some cases, aneurysms were seen to decrease in size but remained incompletely thrombosed (group 2 ). In 1 case (Fig 2), the ophthalmic artery that was originally covered by the PED was later exposed directly to blood flow at 6 months due to device retraction. The demographics of these cases are included in the On-line Table.

The temporal PED changes were observed in group 1 (Fig 3), with retraction at either the proximal or distal end. In these cases, most strands in the device had moved during the retraction, likely altering the metal coverage at the neck of the aneurysm.

Similar results were seen in group 2 as well (Fig 4). By the 6-month follow-up, the arterial curvature distal to the aneurysm was frequently altered by device implantation. In both cases (Fig 


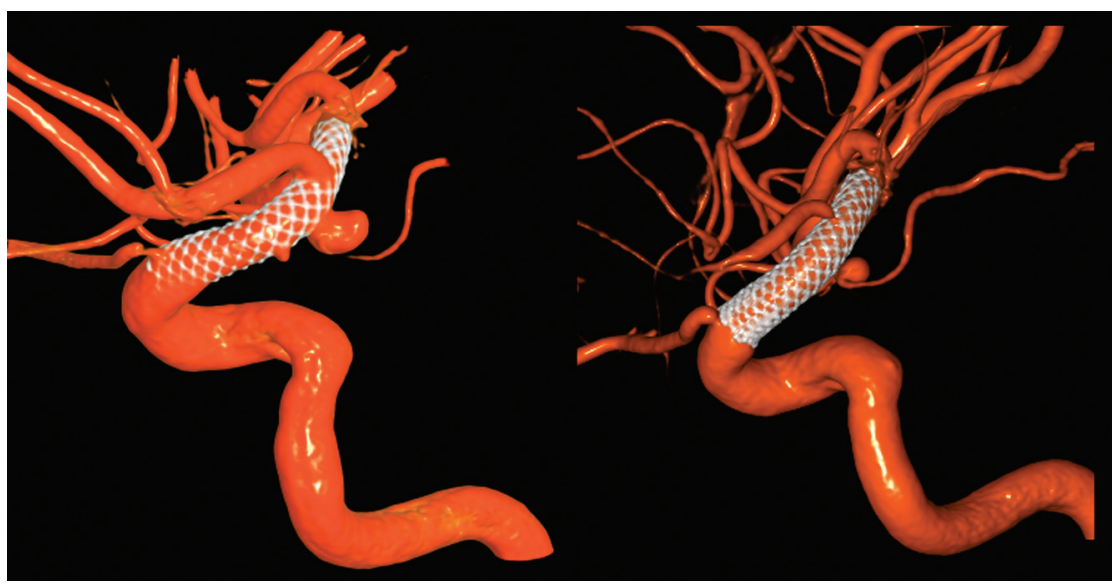

FIG 2. A PED covered the ophthalmic artery initially after treatment, but the PED retracted distally and uncovered the ophthalmic artery at 6 months (patient 7 in group 2).
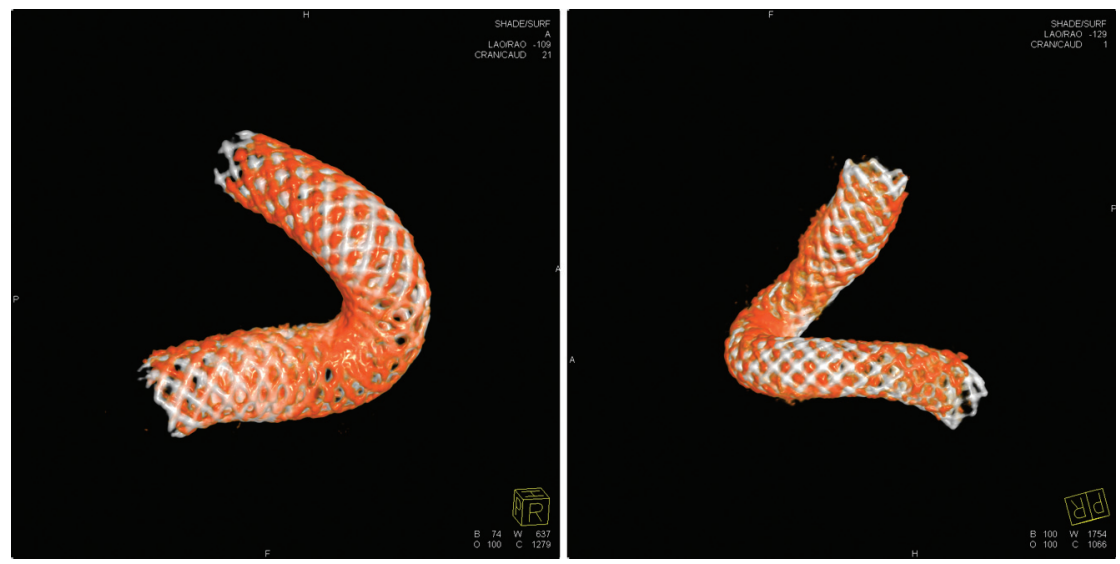

FIG 3. Minor PED retraction is shown in 2 cases in group 1 . The PEDs in white are seen immediately after deployment, and the PEDs in red are from the follow-up, (left) case 2 and (right) case 1.

$4 A,-B)$, aneurysms were located at the region where the greatest deformation occurred. The deployed length was 12 and $18 \mathrm{~mm}$ longer than the nominal length for these 2 cases, respectively, more than $60 \%$ longer than their nominal lengths. By 24-month postdeployment, further deformation of the PED could be observed (Fig 4C); however, the deformation was mostly dominated by the curvature change.

Subtle PED deformation was also observed at both the acute and subacute levels. Fig 5 documents both the acute and subacute changes for an 11-mm supraclinoid ICA aneurysm. This patient was first treated for the aneurysm and then imaged 1 month later when another aneurysm at the contralateral ICA was treated. The proximal end of the PED retracted slightly in the first month (acute change), followed by the proximal strands creeping upward later (subacute change). These changes did not involve the aneurysm neck, and the aneurysm was completely obliterated by 7 months. A well-apposed flow diverter should permit no relative movement between the device and artery. The distal part of the PED in this case was well-apposed to the vessel wall; however, the proximal strands gradually moved distally. This strand-creeping indicated a loose wall apposition and a possible gap between the PED and vessel wall. It is hypothesized that because the aneurysm was located near the distal end of the PED, where the wall apposition had been better, the aneurysm was fully occluded by 7 months.

The Table lists the dimensions of the PEDs immediately after deployment and at the 6-month follow-up. The device oversize is defined as the difference between the nominal diameter and the average of the distal and proximal diameters of a deployed PED, while the PED elongation describes the difference between the deployed PED length and its nominal length. In this study, the PED shortened on average $8.4 \%$ and $11.6 \%$ after 6 months for groups 1 and 2, respectively. The PED also enlarged 0.23 $\mathrm{mm}$ on average for both groups (recoil). The PED remodeling did not appear to be a major factor in determining treatment outcome at 6 months, but the outcome was affected by excessive PED elongation. The difference in elongation (both in magnitude and percentage) between the 2 groups was statistically significant. In cases with considerable PED shortening, the length of the artery remained the same from our image analysis of 3D DSA.

The length of the PEDs at the time of follow-up was closely related to the original deployed length, but the elongation was not affected by the device oversizing, implying multiple mechanisms for device elongation (Fig 6). Furthermore, device oversizing did not influence the shortening of the PED during the first 6 months after treatment, nor did it affect the PED recoil during the same period (Fig 7). The tapering of the ICA compelled the choice of a larger device. The diameter of the ICA decreased by $0.03 \mathrm{~mm}$ for every $1 \mathrm{~mm}$ of length (Table), so for the treated section of an artery $30 \mathrm{~mm}$ long, one could expect the artery to be almost $1 \mathrm{~mm}$ smaller distally, stretching the PED even more and exaggerating the oversizing effects. This level of vessel tapering is not unique to our experience; Fischer et $\mathrm{al}^{15}$ reported a $0.7-\mathrm{mm}$ difference between the proximal and distal diameters in their study of 101 aneurysms.

\section{DISCUSSION}

The advancement of C-arm CT technology permits visualization of radio-opaque strands in the PED at a reasonable resolution, and the entire PED structure can be reconstructed from these images. Our study on the change of PED devices with time challenges the notion that the PED configuration can be predicted by a computer program and that once the PED is implanted, it remains the same permanently. ${ }^{16}$ Deformation of the PED and wall apposition are often overlooked on 2D DSA. Our study demonstrates that a deployed PED involves a dynamic remodeling pro- 

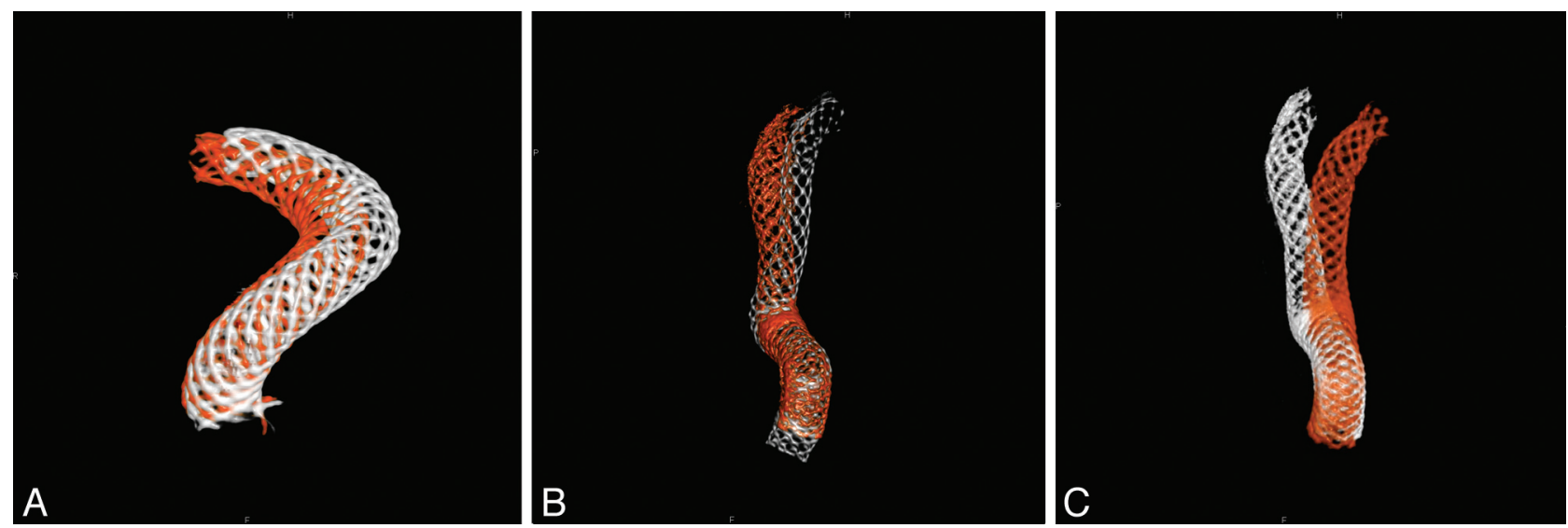

FIG 4. A change of vessel curvature is observed in 2 cases in group 2. A, Case 6 (white is for the posttreatment, and red for 6-month follow-up). $B$, Case 1 (white indicates posttreatment, and red shows the 6-month follow-up). C, Further PED movement in case 1 is observed between 6 (in white) and 24 (in red) months.
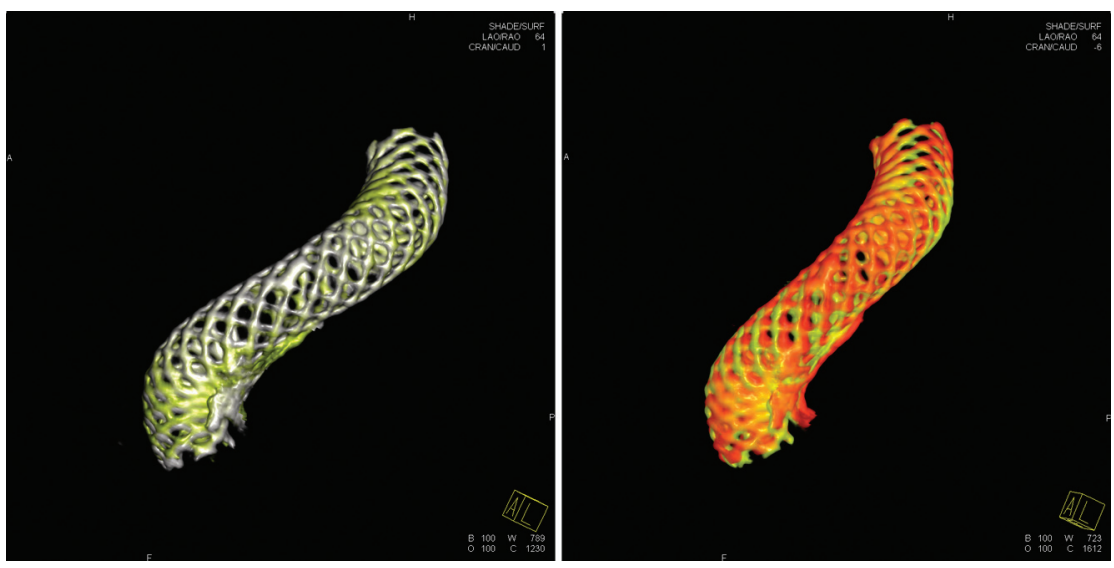

FIG 5. Acute and subacute changes of a $4 \times 18 \mathrm{~mm}$ PED. The PED in white is immediately after deployment, yellow is the 1-month follow-up, and red is the 7-month follow-up. Left: Acute change in the first month after deployment. Right: Subacute change of the PED between the 1and 6-month follow-ups.

\section{Dimensions and measurements of the PED ${ }^{\mathrm{a}}$}

\begin{tabular}{lccc}
\hline & $\begin{array}{c}\text { Occlusion } \\
(\boldsymbol{n}=7)\end{array}$ & $\begin{array}{c}\text { Incomplete } \\
(\boldsymbol{n}=7)\end{array}$ & P Value \\
\hline Nominal PED diameter $(\mathrm{mm}), \mathrm{D}_{0}$ & $4.04(0.45)$ & $4.46(0.51)$ & \\
Nominal PED length $(\mathrm{mm}), \mathrm{L}_{0}$ & $17.43(2.32)$ & $19.43(4.98)$ & \\
Proximal diameter post-Tx $(\mathrm{mm}), \mathrm{D}_{1}$ & $3.77(0.37)$ & $4.13(0.36)$ & \\
Distal diameter post-Tx $(\mathrm{mm}), \mathrm{D}_{2}$ & $3.12(0.45)$ & $3.23(0.52)$ & \\
Deployed length $(\mathrm{mm}), \mathrm{L}_{1}$ & $24.27(5.42)$ & $32.57(8.89)$ & \\
$\mathrm{D}_{1}$ at 6 months $(\mathrm{mm})$ & $3.93(0.33)$ & $4.39(0.28)$ & \\
$\mathrm{D}_{2}$ at 6 months $(\mathrm{mm})$ & $3.32(0.65)$ & $3.51(0.55)$ & \\
Length at 6 months $(\mathrm{mm}), \mathrm{L}_{2}$ & $22.31(5.73)$ & $28.77(7.81)$ & \\
Oversize post-Tx $(\mathrm{mm}), \mathrm{D}_{0}-\left(\mathrm{D}_{1}+\mathrm{D}_{2}\right) / 2$ & $0.59(0.25)$ & $0.78(0.33)$ & \\
Recoil at 6 months $(\mathrm{mm}), \Delta\left(\mathrm{D}_{1}+\mathrm{D}_{2}\right) / 2$ & $0.18(0.19)$ & $0.28(0.18)$ & \\
Elongation $(\mathrm{mm}), \mathrm{L}_{1}-\mathrm{L}_{0}$ & $6.84(4.28)$ & $13.14(4.30)$ & .026 \\
Elongation in \%, $\left(\mathrm{L}_{1}-\mathrm{L}_{0}\right) / \mathrm{L}_{0}$ & $39.29(22.42)$ & $66.68(13.67)$ & .025 \\
Shortening in 6 months $(\mathrm{mm}), \mathrm{L}_{1}-\mathrm{L}_{2}$ & $1.96(1.86)$ & $3.80(2.14)$ & \\
\hline Note- & & & \\
\hline
\end{tabular}

\section{Note:-Tx indicates treatment}

${ }^{\text {a }} \mathrm{SDs}$ are given in parentheses.

cess in which strands may deform individually or together and acutely or with time. As a result, the intra-aneurysmal flow may be evolving. A slight PED retraction demands adjustment of all the strands at least locally (Fig 5); the level of adjustment is greater at the convex side at which the aneurysm is often located than at the concave side where strands are close to each other. Our observation is different from the previously reported delayed migration that may lead to complications or subarachnoid hemorrhage. ${ }^{14}$

Device oversizing is a result of our intention to maintain a proper wall apposition throughout the device, complicated by the tapered ICA. Although in some instances, oversizing has been shown to reduce the therapeutic benefit of flow diversion in hemodynamic simulations, ${ }^{17}$ few studies have examined its potential effect on the aneurysm occlusion rate. There was a difference in the oversize between groups 1 and 2 in our study ( 0.59 versus $0.78 \mathrm{~mm})$, but this difference was not statistically significant. Instead, the oversize was manifested in device elongation. When an oversized PED is selected, the deployed PED tends to be longer than its nominal length because it cannot fully expand. This difference, in turn, will lower the metal coverage over the aneurysm neck, raising the local porosity and causing a potential loss of therapeutic impact of a flow diverter. Thus, while the difference in oversize is rather subtle $(\sim 0.19 \mathrm{~mm})$, the deployed PED length is significantly different between the 2 groups (6.84 versus $13.14 \mathrm{~mm}$ ). A long PED in a highly tapered artery will result in an even longer deployed length, so the selection of the PED length cannot be overlooked.

There are 2 mechanisms by which a PED may remodel, changing its shape and structure in response to the external environment. First, the device could retract at either or both ends. As the retraction progresses, the proximal or distal end enlarges, shortening the device in the meantime and shifting all the strands in between. The PED shortening due to this mechanism is predictable. The second mechanism is the curvature 

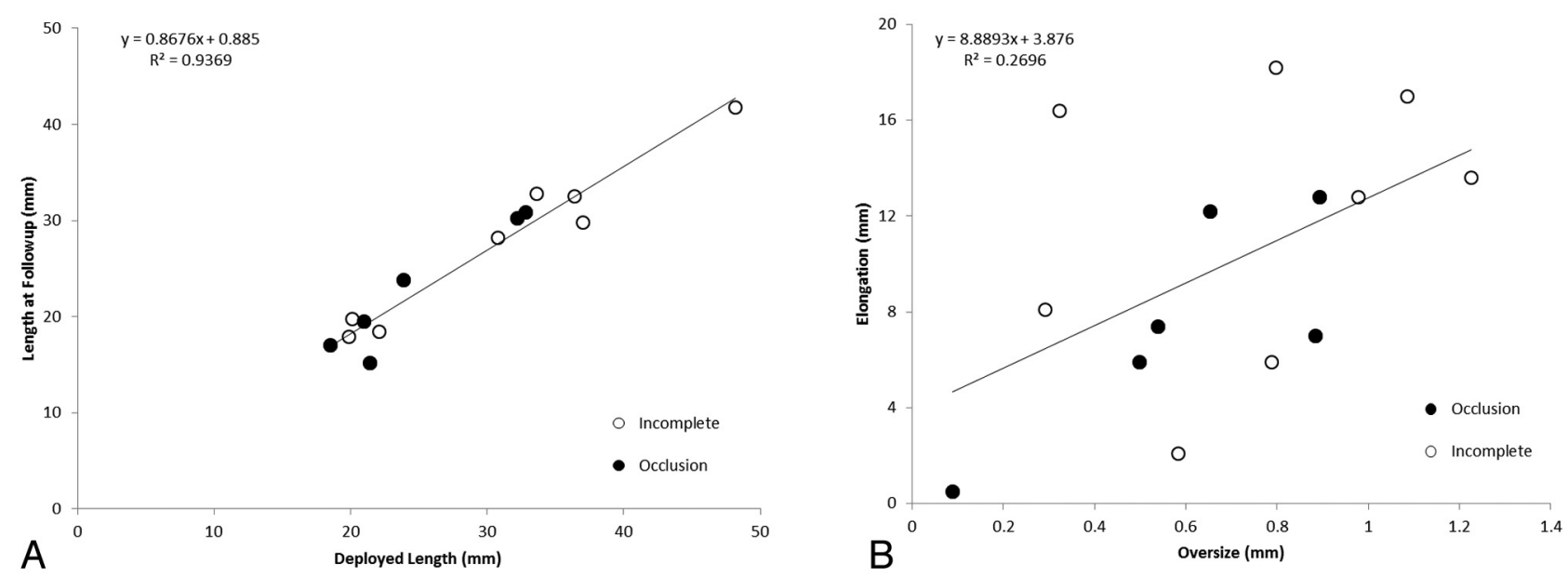

FIG 6. A, A linear relationship between the deployment length and the length at 6-month follow-up. B, The PED elongation is independent of the initial PED oversize.
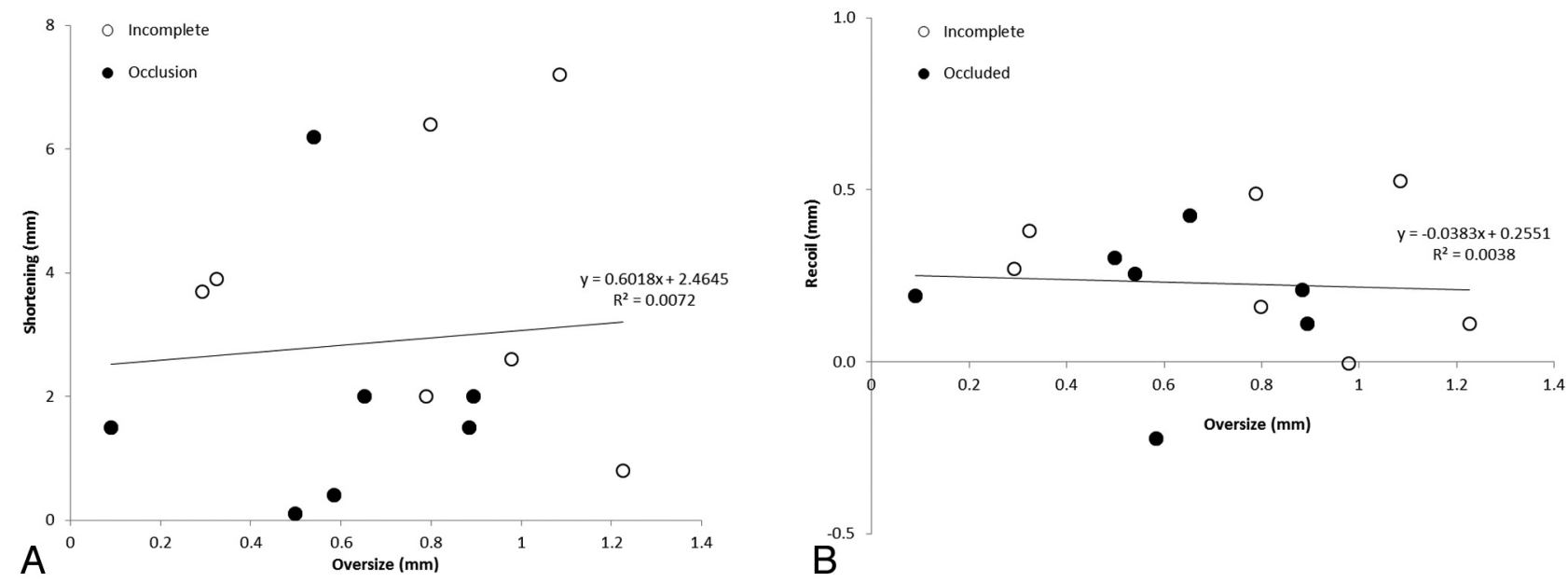

FIG 7. The relationship between device oversize and shortening $(A)$ and recoil $(B)$.

change of the PED with time in response to bending. This phenomenon is commonly seen in cases with other intracranial stents when the artery of interest is tortuous. ${ }^{9,12,18}$ For most cases, the PED remodeling involves both mechanisms, resulting in a complicated relationship between the oversize/elongation (action) and shortening/recoil (reaction). The PED remodeling process becomes even more complicated when 2 PEDs are overlapped or telescoping for treatment of wide-neck or fusiform aneurysms. The first PED device deployed is underneath the others and is highly constrained, and the subsequent PEDs are subject to a different environment. A case of multiple PEDs is included as an example in the On-line Figure in which the proximal PED experienced unexpected and abnormal deformation.

The metal coverage and porosity may evolve with time due to this PED remodeling process. Considering that most PEDs in our studies become shorter and larger with time, the metal coverage will increase at the aneurysm neck, lowering the local porosity. This remodeling brings the device closer to its nominal dimensions, at which the porosity is lower; therefore, the remodeling could be beneficial to aneurysm embolization. Elongation of a PED, however, complicates its deployment. An oversized PED leads to a longer and unpredictable deployed length, challenging the positioning of a PED relative to the aneurysm and neighboring branches. A sufficient overhang is recommended so the aneurysm neck is well-covered should the PED retract. ${ }^{15}$

Braided stents permit a relative motion between the strands, ${ }^{19}$ and friction between the strands is not sufficiently great enough to prevent this motion. ${ }^{20}$ Consequently, individual strands deform, and the PED remodels. Wang and Ravi-Chandar ${ }^{20}$ have developed a mathematic model that describes the mechanics of braided stents. The radial force for a braided stent increases linearly with device oversizing initially. From their model, we estimate that the contact pressure between the PED and artery increases $5 \mathrm{~mm} \mathrm{Hg}$ for every $0.5 \mathrm{~mm}$ of oversize. This is equivalent to a radial force of $2 \mathrm{mN} / \mathrm{mm}$ at the nominal diameter, and 4 and $5 \mathrm{mN} / \mathrm{mm}$ for a 0.5 - and 1- mm oversize, respectively. ${ }^{15,19}$ Thus, a 0.5 -mm oversize doubles the radial force, and any additional oversize produces little or no advantage.

An artery interacts with an implant both biologically and mechanically. The acute elastic recoil, resulting in a smaller lumen, ranges from $9 \%$ to $21 \%$ after deployment of a balloon-expandable or self-expanding stent. ${ }^{21,22}$ This stent recoil is attributed to the elasticity of plaque/vessel and plastic deformation of the (nitinol or stainless) stent during deployment, independent of the size or 
design of stent. ${ }^{22}$ The stent recoil leads to a suboptimal lumen size and possible restenosis; however, this recoil does subside with time (38\% at 8 weeks and $12 \%$ at 6 months). ${ }^{23,24}$ The PED remodeling reduces the forces (and strain energy) required to hold all the strands together and seems to diminish with time in our limited number of cases. Bending of a PED, however, constrains the device and limits how the device can remodel. The device is allowed to adjust only at the section that is not subject to bending. If this process fails, then the adjustment of local arterial curvature is required.

We have treated $>100$ patients with flow diverters since the approval of the PED. However, only a limited number of patients were treated with a single PED, and this contributes to the small sample size in this study. The average number of PEDs used for an aneurysm in the literature is $1.14-2.0,,^{2,4-26}$ and this is very similar to our experience with 1.8 PEDs per case in the first 100 cases. Fifty-three percent of the patients at our institution received 1 PED, compared with 34\%-69\% at other centers, ${ }^{15,25,26}$ and these aneurysms were, in general, smaller than aneurysms treated by multiple PEDs (6.2 versus $12.2 \mathrm{~mm}$ ). Additional PEDs may lower the porosity and reduce the intra-aneurysmal flow simultaneously, rendering rapid aneurysm occlusion. However, the decision to implant multiple PEDs is subjective. Overlapped PEDs are less appreciated on DynaCT images, and interaction among PED devices is quite complicated. Because we have very limited knowledge on the long-term behavior of these flow diverters, the current study is only the first step toward an understanding of the flow diverter interacting with the vessel wall.

Our study is limited by the resolution of DynaCT images, which is by no means close to the dimension of the struts in a PED. However, it does not require a $\sim 30-\mu \mathrm{m}$ resolution for detection of the PED remodeling, ${ }^{27}$ and the effect of PED remodeling is well-demonstrated in the study. The shortening of a PED is as large as 6-7 $\mathrm{mm}$, twice the local diameter of the ICA, and the increase in the circumference is $0.65 \mathrm{~mm}$ on average. These changes are easily seen even without additional imaging postprocessing.

\section{CONCLUSIONS}

Not only does a PED reconstruct the aneurysm and parent artery, but its entire structure goes through a gradual remodeling process. The relative deformation between the PED and the artery indicates suboptimal wall apposition. Device oversizing does not have a direct effect on the shortening or recoil. The aneurysm occlusion rate, however, is lowered by overelongation of the PED.

Disclosures: Liang-Der Jou—UNRELATED: Grants/Grants Pending: Siemens. ${ }^{*}$ Michel E. Mawad-UNRELATED: Board Membership: member of the Scientific Advisory Board at Siemens Medical Solutions, ${ }^{*}$ Consultancy: MicroVention/Terumo, Codman Neurovascular, Payment for Lectures (including service on Speakers Bureaus): MicroVention/Terumo, Stock/Stock Options: Surpass Inc, Other: educational grants to the institution for an educational meeting (Live Interventional Neuroradiology Course Houston) from Siemens Medical Solutions, MicroVention/Terumo, Stryker, Codman Neurovascular, Penumbra. * *Money paid to the institution.

\section{REFERENCES}

1. Crobeddu E, Lanzino G, Kallmes DF, et al. Marked decrease in coil and stent utilization following introduction of flow diversion technology. J Neurointerv Surg 2013;5:351-53

2. Kan P, Siddiqui AH, Veznedaroglu E, et al. Early postmarket results after treatment of intracranial aneurysms with the Pipeline embo- lization device: a U.S. multicenter experience. Neurosurgery 2012;71: 1080-87, discussion 1087-88

3. McAuliffe $\mathrm{W}$, Wycoco V, Rice $\mathrm{H}$, et al. Immediate and midterm results following treatment of unruptured intracranial aneurysms with the Pipeline embolization device. AJNR Am J Neuroradiol 2012;33:164-70

4. Saatci I, Yavuz K, Ozer C, et al. Treatment of intracranial aneurysms using the Pipeline flow-diverter embolization device: a single-center experience with long-term follow-up results. AJNR Am J Neuroradiol 2012;33:1436-46

5. Nelson PK, Lylyk P, Szikora I, et al. The Pipeline embolization device for the intracranial treatment of aneurysms trial. AJNR Am J Neuroradiol 2011;32:34-40

6. Yu SC, Kwok CK, Cheng PW, et al. Intracranial aneurysms: midterm outcome of Pipeline embolization device-a prospective study in 143 patients with 178 aneurysms. Radiology 2012;265:893-901

7. Kulcsár Z, Houdart E, Bonafe A, et al. Intra-aneurysmal thrombosis as a possible cause of delayed aneurysm rupture after flow-diversion treatment. AJNR Am J Neuroradiol 2011;32:20-25

8. Cruz JP, Chow M, O'Kelly C, et al. Delayed ipsilateral parenchymal hemorrhage following flow diversion for the treatment of anterior circulation aneurysms. AJNR Am J Neuroradiol 2012;33:603-08

9. King RM, Chueh JY, van der Bom IM, et al. The effect of intracranial stent implantation on the curvature of the cerebrovasculature. AJNR Am J Neuroradiol 2012;33:1657-62

10. Bing F, Darsaut TE, Salazkin I, et al. Stents and flow diverters in the treatment of aneurysms: device deformation in vivo may alter porosity and impact efficacy. Neuroradiology 2013;55:85-92

11. Clarençon F, Piotin M, Pistocchi S, et al. Evaluation of stent visibility by flat panel detector CT in patients treated for intracranial aneurysms. Neuroradiology 2012;54:1121-25

12. Valdivia y Alvarado M, Ebrahimi N, Benndorf G. Study of conformability of the new Leo plus stent to a curved vascular model using flat-panel detector computed tomography (DynaCT). Neurosurgery 2009;64(3 suppl):ons130-34, discussion ons134

13. Heran NS, Song JK, Namba K, et al. The utility of DynaCT in neuroendovascular procedures. AJNR Am J Neuroradiol 2006;27: 330-32

14. Chalouhi N, Tjoumakaris SI, Gonzalez LF, et al. Spontaneous delayed migration/shortening of the Pipeline embolization device: report of 5 cases. AJNR Am J Neuroradiol 2013;34:2326-30

15. Fischer S, Vajda Z, Aguilar Perez M, et al. Pipeline embolization device (PED) for neurovascular reconstruction: initial experience in the treatment of 101 intracranial aneurysms and dissections. Neuroradiology 2012;54:369-82

16. Ma D, Dumont TM, Kosukegawa H, et al. High fidelity virtual stenting (HiFiVS) for intracranial aneurysm flow diversion: in vitro and in silico. Ann Biomed Eng 2013;41:2143-56

17. Mut F, Cebral JR. Effects of flow-diverting device oversizing on hemodynamics alteration in cerebral aneurysms. AJNR Am J Neuroradiol 2012;33:2010-16

18. Ebrahimi N, Claus B, Lee CY, et al. Stent conformity in curved vascular models with simulated aneurysm necks using flat-panel CT: an in vitro study. AJNR Am J Neuroradiol 2007;28:823-29

19. Duda SH, Wiskirchen J, Tepe G, et al. Physical properties of endovascular stents: an experimental comparison. J Vasc Interv Radiol 2000;11:645-54

20. Wang R, Ravi-Chandar K. Mechanical response of a metallic aortic stent. Part I. Pressure-diameter relationship. J Appl Mech 2004;71:697-705

21. Aziz S, Morris JL, Perry RA, et al. Stent expansion: a combination of delivery balloon underexpansion and acute stent recoil reduces predicted stent diameter irrespective of reference vessel size. Heart 2007;93:1562-66

22. Danzi G, Fiocca L, Capuano C, et al. Acute stent recoil: in vivo evaluation of different stent designs. Catheter Cardiovasc Interv 2001;52:147-53

23. Tanimoto S, Bruining N, van Domburg RT, et al. Late stent recoil of the 
bioabsorbable everolimus-eluting coronary stent and its relationship with plaque morphology. J Am Coll Cardiol 2008;52:1616-20

24. Berrocal DH, Gonzalez GE, Fernandez A, et al. Effects of overexpansion on stents' recoil, symmetry/asymmetry, and neointimal hyperplasia in aortas of hypercholesterolemic rabbits. Cardiovasc Pathol 2008;17:289-96

25. Colby GP, Lin LM, Gomez JF, et al. Immediate procedural outcomes in 35 consecutive Pipeline embolization cases: a single-center, single-user experience. J Neurointerv Surg 2013;5:237-46
26. Lylyk P, Miranda C, Ceratto R, et al. Curative endovascular reconstruction of cerebral aneurysms with the Pipeline embolization device: the Buenos Aires experience. Neurosurgery 2009;64:632-42, discussion 642-43, quiz N636

27. Ionescu M, Metcalfe RW, Cody D, et al. Spatial resolution limits of multislice computed tomography (MS-CT), C-arm-CT, and flat panel-CT (FP-CT) compared to MicroCT for visualization of a small metallic stent. Acad Radiol 2011;18:866-75 